SCHOLEDGE International Journal of

Management \& Development

ISSN 2394-3378, Vol.06, Issue 4

$\operatorname{Pg} 34-47$.

DOI: 10.19085/journal.sijmd060401
Published by: SCHOLEDGE Publishing

www.theSCHOLEDGE.org

Email: editorial@thescholedge.org

\title{
Will Artificial Intelligence Change Strategic Top Management Competencies?
}

\section{Prof Dr. M S S El Namaki}

Dean, Victoria University, School of Management, Switzerland.

Dean (Retired) Maastricht School of Management, MSM, And The Netherlands.

\section{The Problem}

The probability is high that Artificial Intelligence (AI) will provide the answer to future product and market innovations. It has the ability to induce a fundamental change in products, markets, models, and paradigms. Manufacturing and a wide spectrum of services are undergoing a near-revolutionary change driven by AI delivered innovations. Computing equipment capable of what one may term partial and quasi-intelligent behavior is the trigger. Ways and means of converting this new phenomenon into the strategic behavior of firms is a murky process today. Yet this process will create new premises for strategic thinking and, in the process, new demands on executive competencies.

This will be the focus of this article.

The article explores present-day contours of Artificial Intelligence and provides an attempt at deriving the impact of the phenomenon on the performance of the strategic thinking function within business environments. It proceeds, then, to translate the findings into implications for executive strategic thinking competencies.

The article relies on present-day research on Artificial Intelligence and strategic thinking. It could provide a significant contribution to the restructuring of executive strategic thinking competencies. 


\section{The Artificial Intelligence Concept}

\section{What is Intelligence and What is Artificial Intelligence}

A broad definition equates intelligence to "the ability to acquire and apply knowledge and skills" (Legg and Hutter, 2007). This author subscribes, however, to a Thurstone theory of intelligence that equates intelligence to seven different primary mental abilities. (Louis L. Thurstone: 1943). Those include Verbal comprehension, reasoning, perceptual speed numerical ability, word fluency, associative memory, and spatial visualization. Some of those constitute building stones of artificial intelligence.

Artificial intelligence, on the other hand, is defined by Merriam-Webster as "A branch of computer science dealing with the simulation of intelligent behavior" as well as "The capability of a machine to imitate intelligent human behavior". (Forbes, Feb 14, 2018,). In other words, AI is the simulation of human intelligence processes by machines, especially computer systems. These processes include learning (the acquisition of information and rules for using the information), reasoning (using the rules to reach approximate or definite conclusions) and self-correction. AI relates to many sciences from computing and data to psychology, philosophy, and linguistics among others.

\section{AI as a System}

To an entrant, Artificial Intelligence (AI) constitutes an amorphous phenomenon. A wide array of concepts, sub-concepts, and hypotheses are presented. And an equally wide array of terms, labels and at times jargon are thrust. Technologies are touted and ultimate outcomes are mystified. It looks like an industry in the early stages of a life cycle with the life cycle itself shrouded into the futuristic mystery.

One way of structuring this whole is to put it within a systems theory framework. AI is then viewed as a process where inputs go through a transformation mechanism that leads to output and a feedback loop.

\section{Phases of Artificial Intelligence.}

There are different approaches to AI technology segmentation. One can consider AI in terms of time i.e. present day vs. future events, or state of complexity i.e. weak or strong or degree of "genericness" i.e. General AI vs. Applied AI. The author opts for the time dimension which segments AI into present day versus future (Hintze, 2016).

\section{- The Present}

Present day intelligent systems are systems able to handle massive volumes of data but lack the analytical and independent self-awareness element. They are either reactive or limited memory. 
- Reactive. These are equipment that analyzes possible moves, their own and their opponent's, and choose the most strategic move. They do not have the ability either to form memories or to use past experiences in order to guide current decisions. The computer's perception of the world is direct and it acts according to what it "sees".

- Limited memory (corrective). These equipment use past experience in order to influence future decisions. Past information is, however, only transient and are not saved as part of a library or a learning experience. (The Conversation, November 14, 2016).

\section{○ The Future.}

This AI segment does not only form an image of its own world but also of other agents or entities in the world at large. It does not only understand consciousness but has it.

- Theory of mind. This is a psychology term that refers to the ability to attribute mental states, beliefs, knowledge, desires, and intentions, to oneself and to understand that others have beliefs, knowledge desires and intentions that are different from one's own. (Baron, Simon 1991) and are of impact on their decisions. This kind of AI does not yet exist.

- Self-awareness. In this category, AI systems have a sense of self and consciousness. Machines with self-awareness understand their current state and can use the information to infer what others are feeling. Conscious beings are aware of themselves, know about their internal states, and are able to predict feelings of others. This type of AI does not exist yet. (The Conversation, November 14, 2016)

\section{The Human Brain Simulation Dimension.}

Artificial intelligence connotes a process of simulation of the human brain. Key constituents of the human brain are a" CNS" or "Central Nervous System" built around brain cells and a spinal cord and a "Periphery Neural System" "or" PNS" made up of sensory neurons and motor neurons. Two essential features of the system are the functions of the components and the mode of communication. Neurons are either sensorbased or motor-based. Sensor neurons scan the immediate and distant environments for signals. A motor neuron responds to a signal from the Sensor Neuron or the inter-neurons node (within the CNS) by issuing instructions to an organ or a functional instrument.

This system could constitute a simulation of the human brain. The brain .collects data and operates through neurons or nerve cells. Information flows from one neuron to another across a small gap called a synapse (SIN-aps). At the synapse, electrical signals are translated into chemical signals in order to cross the gap. One sending neuron can connect to several receiving neurons, and one receiving neuron can connect to several sending neurons (Stufflebeam, 2008). (Genetic Science Learning Center 2015, June 30.) 
Artificial neural networks a system of hardware or software that is patterned after the working of neurons in the human brain and nervous system

Figure (1): Communications within a biological neuron network: Neurons transmit messages in the brain.

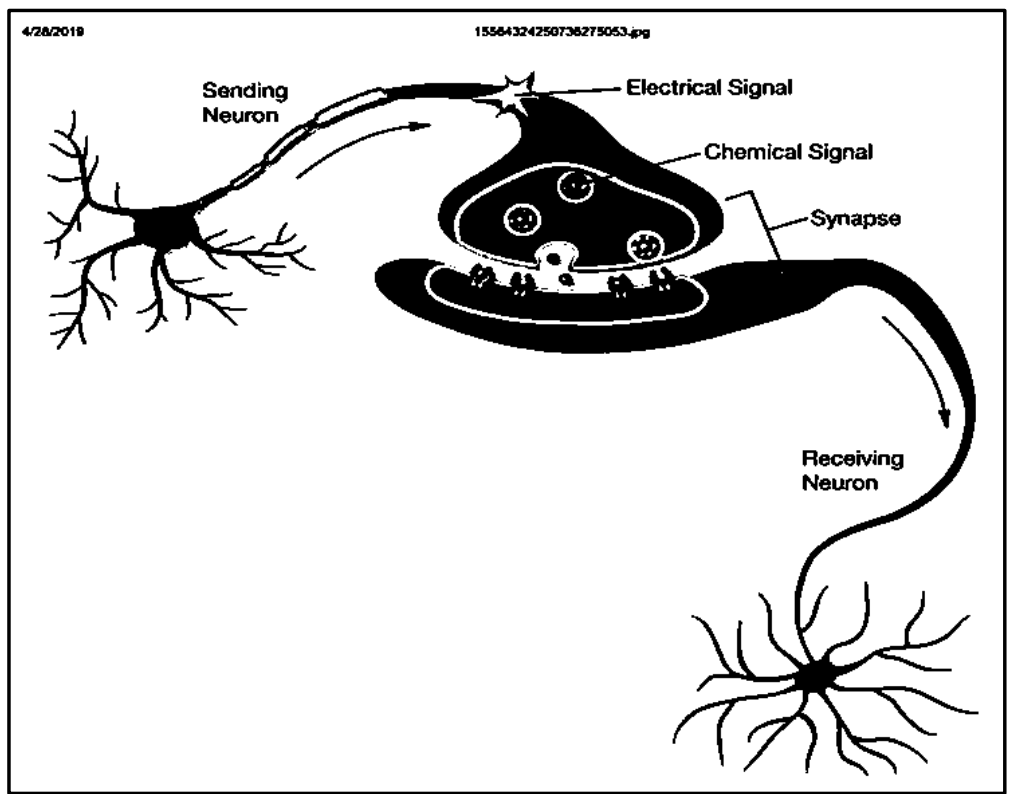

Source: Genetic Science Learning Center. (2015, June 30)

\section{What does all of that mean for Strategic Thinking?}

Artificial intelligence modes and instruments have been adopted in industry, services, and even agriculture. The adoption reveals a powerful potential and an impending long term impact. It is more likely than not to trigger shifts in the framework, the processes, and the players. Consider the following cases.

\section{Case one:}

\section{Tangli's Smart Cities and Digital Platform}

Artificial Intelligence is helping to address urban challenges by enhancing the effectiveness of city and urban management. Both Tangli and Huawei developed a smart city digital platform utilizing artificial intelligence (AI) and Internet of Things (IoT) capabilities in order to manage public safety, environmental protection, transportation, government, education, and agriculture. Huawei's digital platform connects what it calls the brain, the central nervous system, and a peripheral nervous system, made up of sensors across a city. The platform is compatible with different city sensors, creating a city digital twin, and supporting diverse city applications. TANGLI, a Chinese data science operator, has also developed a similar smart city formula. It is similar to Huawei 
in terms of the resort to sensors to collect data that is later subjected to AI analyses with an outcome put to use in urban city management.

\section{Case two:}

\section{Beyer, Pharma and PVAI}

The pharmaceutical industry is set to benefit from AI in a wide number of areas from drug discovery and testing to drug manufacturing, diagnostic assistance, medical treatment process, and application monitoring. Bayer, the German pharma maker, is enhancing its patient safety data monitoring by resorting to AI in an attempt at early tracing of drugrelated side effects. Pharmacovigilance Artificial Intelligence (PVAI) or the PVAI solution, which extracts adverse event data from source documents in an automated fashion, has taken part into and won, a number of competitive proof-of-concept trials run by several large pharmaceutical companies. The ability to apply advanced AI technologies, with the significant process and domain expertise, will usher in a whole new era of pharmacovigilance. It will serve as a single machine learning system that can be utilized across the healthcare industry by life sciences companies, healthcare providers, and regulators. (Pharmaphorum, Nov 2, 2018).

\section{Case three:}

\section{NATO, Defense and AICA}

$\mathrm{AI}$ is changing the very nature of the defense industry with hardware rapidly taking a second place to the software. NATO research points to intelligent, partly autonomous agents becoming primary cyber fighters on future battlefields. Those intelligent software agents would perform, then, active, largely autonomous cyber defense actions within military computerized networks. A situation is envisaged where enemy software cyber agents-malware-would infiltrate friendly networks and attack friendly computerized command, control, communications, intelligence, surveillance, and reconnaissance weapon systems. To fight this hostile "software", NATO is developing artificial cyber huntersintelligent, autonomous, mobile agents specialized in active cyber defense. That Autonomous Intelligent Cyber Defense Agents (AICA) is more likely than not to be the product of AI research. (ARL: March 2018) (Kott, March 2018). 
Figure (2): Artificial Intelligence and Army Innovation

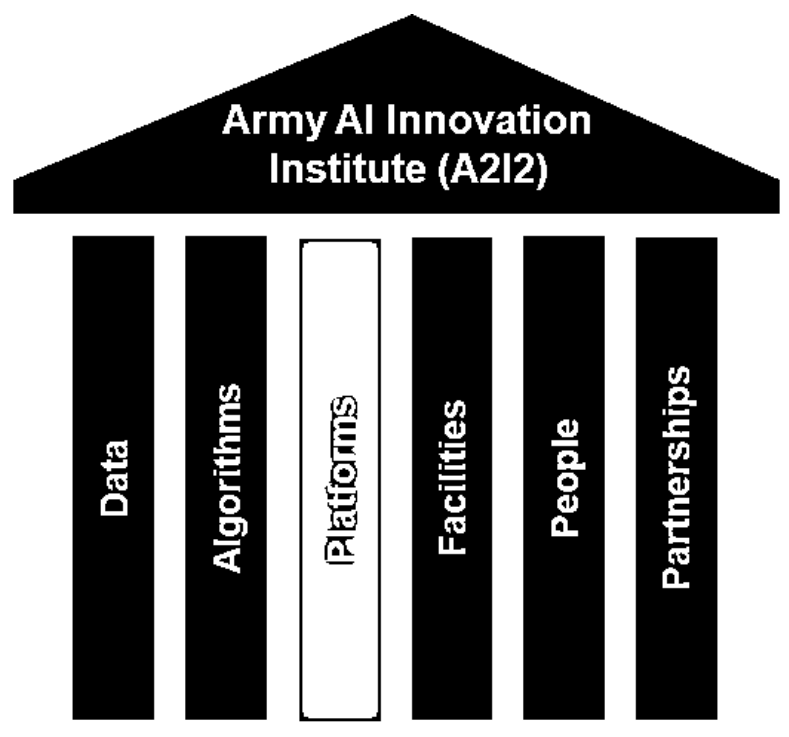

Source: Prevailing in a Complex World: ARL's Essential Research Area on AI \& ML,

Case four

Huawei and the G5 Technology

Four key technologies are converging in order to transform the Information and Communications Technology (ICT) ecosystem. Those technologies include the fifth generation wireless communications technology $(5 \mathrm{G})$ and the Internet of Things (IoT). Huawei is a key player in this endeavor. Super-speed "fifth-generation" (5G) mobilephone networks will require heavy upgrading investment by telecom firms all around the World. Huawei-along with rivals such as Ericsson and Nokia, - are competing for the prime role as the main player and principal supplier. G5 provides a vital enabling technology for the "Internet of Things", in which all manner of devices, from cars and industrial robots to toasters and children toys, will talk to each other, and to the internet at large. And Huawei, again, is a front runner. (Economist, Dec 7th, 2018). 


\section{Observed and Potential Shifts in the Premises of Strategic Thinking.}

Case events cited above could lead to a tentative set of hypotheses of AI impact on the strategic thinking process and the possible shifts. The following are five key hypotheses touching five major areas of potential change.

\section{From Strategic Management to Strategic Innovation}

AI will change the essence of strategic thinking and the process of strategy formulation.

A shift will take place from strategic management to strategic innovation. Data-driven information flows and probabilities used to address uncertainties will create new business paradigms. Traditional operating models, roles, competencies, structures and measures will, as a result, change. Conventional management strategy formulation processes involving event planning, resource assignment, executive action taking, etc. will give way to new modes and approaches redefining strategic behavior, competitive profiles and measures of fulfillment (Information Age, Oct 2018). The static notion of organizations will give way to a dynamic posture where organizations become variables. An organization will exist because it fulfills a product or service-related function but technology will change those function fulfilling instruments and the organization will live or die with this change. Blackberry is a case in point. The technology-driven shift from keyboard entry instrument to touch screens led to an all-inclusive restructuring of the organization.

\section{From Product to Function.}

AI frameworks will lead to a shift from strategic product and market focus to function focus.

Functions will determine the instrument, being a product or a service, congruent with the changing technology and business conditions. Function analysis derived from big data will contrast with 'need analysis" drawn from market parameters. (Mirko Karakašić et al, 23, 5 (2016)) Rather than relying on customers to tell a business what they want from a product, data analysis will point to the ultimate function fulfilling medium. IBM Watson software is a case in point. The software feeds online consumer preference digital bits of information into an AI construct that helps bring curated shopping journeys to mass audiences. The software, known as GWYN, provides a range of order management and customer engagement capabilities to e-commerce retailers through information provided by consumers about a gift recipient (Techno emergence Oct 28, 2018). 


\section{From Time as a Yardstick to Events as a Measure.}

AI technologies will substitute time as a standard of fulfillment by event completion as a measure.

Conventional managerial thinking resorts to the labeling of time as a term and projecting this term according to duration i.e. short, medium or long terms. Goals, actions or events are made a function of this term taxonomy. AI will set event completion as a standard. Instant events and distant events will mark the passage of time and replace the short and long term convention. Duration of events and the intervals between them will mark motion. Event flow and sequence will constitute a yardstick to measure business strategies and their ultimate outcome. This concept is already taking hold. Consider the current race for 5G technology. The United States is measuring the event in terms of Huawei's ability to introduce technology across the globe. The conventional long term has disappeared and is replaced by an event i.e. the entry into force of $5 \mathrm{G}$ technology applications. One key goal of $5 \mathrm{G}$ is to dramatically improve quality of service, and extend that quality over a broader geographic area within an instant event framework.

\section{From Industry to Arena.}

AI learning and data analytics will introduce fundamental shifts in the concept of industry.

Long cherished industry structure parameters as the power of buyers, power of suppliers, substitution, entry, and rivalry (Porter, 1980) will give way to factors as industry function fit, industry technology life cycle, industry overlap and industry downfall. New industries will, as a result, replace old ones. Health sciences, financial services, communication, energy, and retailing industries are all going through this process. Some are crossing traditional industry boundaries and merging or substituting others while a few are showing signs of decline and eventual death. Financial services illustrate this phenomenon. The financial service industry has been adopting technologies like cloud computing and voice recognition and changing the very essence of the service. Meanwhile, technology operators are moving in the opposite direction by incorporating financial services into their technology mix. The outcome is an ever-narrowing gap between finance and technology. Actually, competition is emerging from large, established technology companies like Google and financial institutions as Citibank. (Forbes, Feb 14, 2019,)

\section{From Competitive Advantage to Competitive Intelligence}

Future competition among firms will depend on innovative intelligence rather than a situational advantage. 
Concepts of the business competition will assume different parameters from those cited in contemporary economics and management frameworks. Data will constitute a pivot here. Businesses extracting "intelligence" from data will have an edge within an environment driven by artificial intelligence forces. Quality of this intelligence will make the difference between competing firms. Data analytics will allow industry leaders to launch entirely new business models and industrial arenas. They will be supported by a multiple of technologies from new types of data sets ("orthogonal data") and massive data integration capabilities to hyper-scale digital platforms. Systems enabled by machine learning can provide a unique competitive advantage in areas as varied as customer service and logistics. Data and analytics can enable faster and more innovative strategies and evidence-based decision making (The age of analytics; competing in a data-driven world, (Mckinsey Dec 2016)

\section{What would that Mean for Top Management Strategic Thinking Competencies?}

It is the author's contention that AI concepts and operations will have a far-reaching disruptive impact on top management strategic thinking and application.

Consider the following.

\section{Adopting Technology as a Strategy Pivot.}

Future top management strategic thinking is more likely than not to be driven by two prime forces: technology and capital (El Namaki, 2014). AI will shape the technology side of the equation. This technology side will constitute a disruptive force that will create imbalance and a rush for company re-positioning within the emerging environments. A prime example is the $(5 \mathrm{G})$ or the fifth generation wireless communications technology which is the latest iteration of cellular technology, engineered to greatly increase the speed and responsiveness of wireless networks. It will enable a sharp increase in the amount of data transmitted over wireless systems due to more available bandwidth. US hostility towards Huawei and before it ZTE is typical for the kind of .top management technology rooted strategic upheaval that corporate leadership can land into...

\section{Conceiving Dynamic Visions.}

Conceiving a vision was, until recently, an orderly process where a vision was regarded as an image of a future state an organization, a corporate culture, technology or activity and the relative position of the organization within that time-space continuum (El namaki, 1992). Conceiving a vision belonged to the heart of the CEO function and constituted a measure of his achievement. Both the vision and the role of the CEO are changing fast. AI complemented with emerging waves of economic and social disruptions is leading to

distinct blur in future images and a measurable difficulty at identifying sharp contours of 
the times to come. This is making static yesterday concepts of vision obsolete. Top management will have to conceive an array of visionary "scenes" or a series of possible positions compatible with the change configuration of technologies, industries, products, processes, and markets. Static visions are being replaced by dynamic images of continuously changing theaters and this will require different CEO competencies from those of yesteryear. Intimacy with the concept of AI and the impact of that concept on functions and product will be a must. Building and leading organizations compatible with the emerging conditions will be another must.

\section{Demonstrating Data Management Competencies}

A competency is an ability to apply knowledge and understand it (Vervenne, 2010). Data analytics competency depends on data quality, data volume, analytical skills, domain knowledge, and tool sophistication (Ghasemaghaei, 2018). Competency at managing data and the consequent processes of data - rooted learning will demand new top management competencies. Subjects as critical thinking and creativity will constitute a critical periphery for strategic thinking. Data analytics will, however, provide the core of the process. Descriptive analytics, diagnostic analytics, predictive analytics, and modular analytics will constitute the pivot of future strategic thinking. Top management will not be able to formulate strategies that match contemporary disruption unless they develop a good understanding and an ability to interact with data. This interaction could relate to the insights that data analysis could reveal, the technologies that data analysis could lead to, the new arenas that data could divulge and the new opportunities that data analysis could unmask. This may require a process of re-education of top management and a realization that yesteryears forces and models may constitute a future peril instead of a boon.

\section{Understanding Novel Analytical Models.}

AI is inducing a change in the conceptual and operational framework of key concepts all the way from strategic design to strategic control. Derived issues as strategic thinking models, leadership traits and demands, industry life cycle will have to reflect grass root shifts triggered by artificial intelligence. This is substantiated by the common feeling today that classic concepts of competitive strategy and organizational behavior are reaching the end of their life cycle. They represent yesteryear's conditions and would very likely fail to reflect the disruptive shifts triggered by new learning and analysis concepts (Ghasemaghaei, 2018).

Forces of disruption are rendering conventional modes of strategic thinking irrelevant. Core concepts as a competitive advantage, the longer term, visions, life cycles, etc. are becoming blurred, at best. The search is for approaches that accommodate the emerging new normal. 


\section{Adopt Neo-Leadership Parameters}

Leadership will lose the generic texture it maintained over the years. Instead, an AI rooted concept will emerge where perspective and control will become the parameters. Perspective is induced by the shifting AI environment and control will measure progression towards new status. A propensity to lead or a measure of an individual's ability to perform a leadership function is likely to emerge. A high propensity is synonymous with a penetrative AI induced perspective and conducive control.

Perspective and control take the issue out of the traditional single-track focus on the leader's behavior or traits to the broader context of the leader, as an individual, the followers, as players and the context, as a disruptive environmental force. Perspective is a reflection of the vision and desire to achieve. Control is a measure of locus and management of self. Analysis of supportive case evidence drawn from Wanda, Blackberry and IKEA lead to the introduction of the Propensity to Lead or a measure of the attributes of those two determinants (El namaki, 2017).

All in all, a thorough process of top management re-education will be essential. This will have far-reaching implications to the current generation of top management executives as well as the emerging generation.

\section{Summary and Conclusions}

The probability is high that Artificial Intelligence (AI) will provide the answer to future product and market innovations. It has the ability to induce a fundamental change in products, markets, models, and paradigms. Manufacturing and a wide spectrum of services are undergoing a near-revolutionary change driven by AI delivered innovations. Computing equipment capable of what one may term partial and quasi-intelligent behavior is the trigger. Ways and means of converting this new phenomenon into the strategic behavior of firms is a murky process today. And how the teaching of strategic thinking will take place under those disruptive conditions is a serious question in need of an answer.

This article addresses this issue. It explores present-day contours of Artificial Intelligence and provides an attempt at deriving the impact of the phenomenon on the performance of the strategic thinking function within business environments. It suggests, based on case analyses, a shift in strategy orientation, time dimension, and leadership framework and industry structure. It proceeds to translate the findings into implications for top management strategy competencies. 


\section{References}

[1]. Thurstone L L, , "Primary Mental Abilities", University of Chicago Press, 1943

[2]. Legg S, Hutter M, "A Collection of Definitions of Intelligence". Jun 25, 2007

[3]. The Key Definitions Of Artificial Intelligence (AI) , Forbes, February 14, 2018 https://www.forbes.com/.../bernardmarr/2018/.../14/the-key-definitions-of-artificial-i

[4]. Hintze A, "Understanding the four types of AI, from reactive robots to self-aware beings", The Conversation, Nov 14, 2016

[5]. Communication between Neurons: Neuroscience in Action, 2014.

[6]. Stufflebeam R, "Neurons, Synapses, Action Potentials, and Neurotransmission", CCSI, 2008

[7]. Neurons Transmit Messages In The Brain. Genetic Science Learning Center. (2015, June 30). https://learn.genetics.utah.edu/content/neuroscience/neurons/

[8]. Digital tool aims to cut costs and risks of underperforming trials, June 6, 2019

[9]. Pham T, Solomon L, Cirincione G and Henz B, "Prevailing in a Complex World: ARL's Essential Research Area on AI \& ML", also Fresconi F et al "ARL experts are on target to find solutions for the future battlespace”, February 9, 2018 https://pharmaphorum.com/news/bayer-artificial-intelligence-pharmacovigilance/

[10]. "Fifth generation, fifth column, why does 5G have everyone worried about Huawei?" Economist, Dec 7th, 2018.

[11]. The age of analytics; competing in a data-driven world, Mckinsey Dec 2016

[12]. El Namaki M, "How Companies are Applying AI to the Business Strategy Formulation" Scholedge International Journal of Business Policy \& Governance Vol.05, Issue 08 (2018).

[13]. El Namaki, M. S. S. (2019), "Conceiving a Vision within Artificial Intelligence Environments", International Journal of Management and Applied Research, Vol. 6, No. 1.

[14]. Scientists Figured Out How to Make Neural Networks 90 Percent Smaller—but Just as Smart, Popular mechanics, May 7, 2019

[15]. El Namaki, M. S. S. (2017), "Disruption and the Changing Concept of Leadership ", International Journal of Management and Applied Research, Vol.4, No. 2,

[16]. El Namaki, M. (1992), Creating corporate vision, Long Range Planning

[17]. Bayer applies artificial intelligence to its pharmacovigilance systems, harmaphorum, November 2, 2018.

[18]. Vervenne L, Najjar J, Ostyn C"Competency Related Data Management (CrDM)", SYNERGETICS, 2010.

[19]. Ghasemaghaei M,Ebrahimi S, Hassanein K ,"Data analytics competency for improving firm decision making performance" The Journal of Strategic Information Systems, Volume 27, Issue 1, March 2018

[20]. BigTech Poses A Greater Threat To Financial Institutions Than FinTech Mayra Rodriguez Valladares, Forbes, Feb 14, 2019,

[21]. The age of analytics; competing in a data driven world, (Mckinsey Dec 2016)

[22]. Porter M (1980) Competitive strategy. 